\title{
Modeling GHS-USD Exchange Rate in Ghana: Application of Stochastic Volatility Model
}

\author{
A.Y. OMARI-SASU \\ Kwame Nkrumah University of Science and Technology, \\ KNUST, Kumasi, Ghana. \\ National Institute for Mathematical Sciences, NIMS, Ghana. \\ Email: ayomari-sasu@knust.edu.gh \\ Tel: +233246289226 \\ REINDORF NARTEY BORKOR \\ Kwame Nkrumah University of Science and Technology, \\ KNUST, Kumasi, Ghana. \\ National Institute for Mathematical Sciences, NIMS, Ghana. \\ Email: reinbork@gmail.com \\ Tel: +233544714398
}

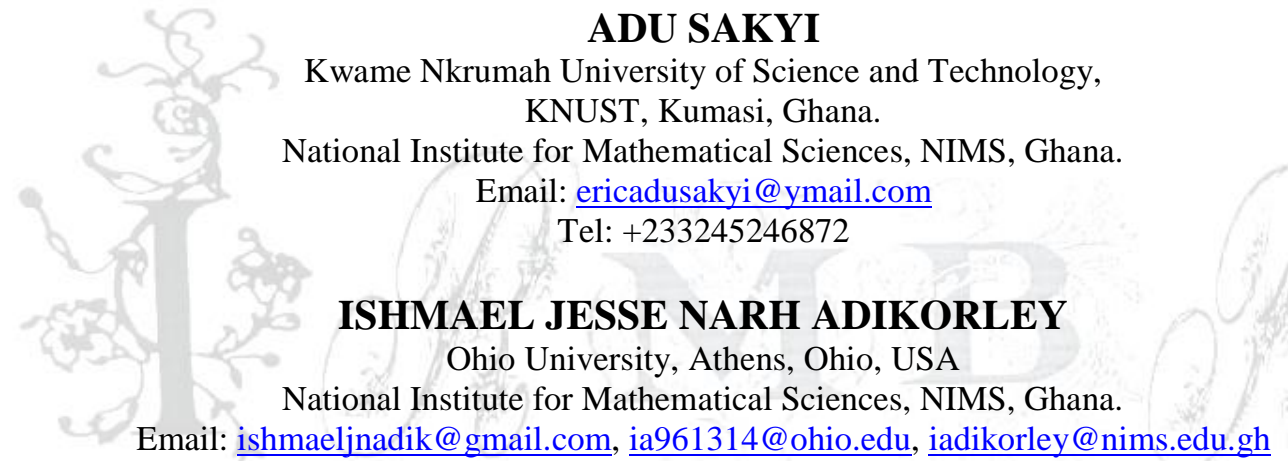

Tel: +17405890753

\begin{abstract}
A very significant input to monetary policymaking is estimating the current level of exchange rate. This paper examined the application of stochastic volatility of returns on the Ghana Cedis and US dollar (\$) exchange rate. Stationarity of the dataset was achieved after differencing. Markov Chain Monte Carlo $(M C M C)$ was used in the parameter estimation. A stochastic volatility (SV) was obtained with the level of log-variance $=\mu$ 10:8320, the persistence of log-variance- $=0: 9285$, and the volatility of log-variance $=$ 0:660. Posterior density estimates, standardized residual plots as well as estimated volatilies were actualized.
\end{abstract}

Keywords: Stochastic Volatility, Markov Chain Monte Carlo (MCMC), GARCH.

\section{Introduction}

Dispersion of asset price returns is measured by volatility. Volatility, being a second moment, characterizes the uncertainties that the market assigns to the price of a particular asset. Volatility refers to the amount of uncertainty or risk about the size of changes in a security's value. A higher volatility means that a security's value can potentially be spread out over a larger range of values. This means that the price of the security 
can change dramatically over a short time period in either direction. A lower volatility means that a security's value does not fluctuate dramatically, but changes in value at a steady pace over a period of time. An alternative approach to describe the volatility evolution of a financial time series is to introduce an innovation to the conditional variance equation, (Melino and Turnbull, 1990), (Taylor, 1986) with the resulting model referred to as a stochastic volatility (SV) model.

Though the difficulty in estimating an SV model is understandable because the model introduces an innovation for each shock, literature shows that SV models often provides improvement in model fitting. Exchange rate volatility is defined as the risk associated with unexpected movements in the Exchange rate. Economic fundamentals such as the inflation rate, interest rate and the balance of payments, which have become more volatile are themselves sources of exchange rate volatility. Exchange rate affects trade volumes, a study by Harvey et al.(2008) looked at the relationship between stock markets and for- eign exchange market and determined whether movements in exchange rates have an effect on stock market in Ghana. It was found that there is negative relationship between exchange rate volatility and stock market returns and a depreciation in the local currency leads to an increase in stock market returns in the long run. Where as in the short run it reduces stock market returns. Prior knowledge on the volatilities of exchange rates does not only help policy makers but individuals as well.

Frimpong et al., (2006) modeled and forecasted volatility (conditional vari- ance) on the Ghana Stock Exchange using a Random Walk, GARCH $(1,1)$, EGARCH $(1,1)$ and TGARCH $(1,1)$ models. They used unique three days a week Databank Stock Index (DSI) to study the dynamics of the Ghana stock market volatility over 10 -years. They estimated the competing volatility models and their specification and forecast performance. They found that the volatility clustering, leptokurtosis and asymmetry effects associated with stock market returns on more advanced stock markets. They estimate that GARCH models suggest a high degree persistent in the conditional volatility of stock returns on the Ghana Stock Exchange.

Suk Joon Byun et al., (2011) investigated whether the superiority of the implied volatility from a stochastic volatility model over the implied volatility on the forecasting performance. They made a grander causality tests between an implied volatility and a realized volatility is carried out for checking the forecasting performance. They implied a trading strategy for forecasting power of an implied volatility earns positively, in particular, more positively under high volatile market or low return market. They identified that as a volatile market grows, it becomes more and more important to evaluate the forecasting performance of intraday future volatility, not only from an academic prospective, but also in practical terms. They suggested that implied volatilities are more meaningful for the future realized volatility.

(Isao Ishida et al.,2011) proposed a new method for estimating continuous- time stochastic volatility models for the SnP 500 stock index process using in- traday high-frequency observations of both the SnP 500 index and the Chicago Board Options Exchange implied volatility index. They provided a framework for using intraday high frequency data of both the indices' estimates, in partic- ular, for improving the estimation accuracy of the leverage parameter driving the diffusive components of the price process and its spot variance process, re- spectively. They experimented the importance of making proper adjustments to the moment conditions when realized measures are computed using data from non-contiguous non-full day trading sessions.

Denis Erdemlioglu et al., (2013) in their paper reviewed the rapid advances in foreign exchange volatility modeling made in the last three decades. According to them, academic researchers have sought to fit the three major characteristics of foreign exchange volatility: intraday periodicity, autocorrelation and discontinuities in prices. Early research modeled the autocorrelation in daily and weekly squared foreign exchange returns with ARCH/GARCH models. Increased com- puting power and availability of high-frequency data allowed later researchers to improve volatility and jumps estimates. Researchers also found it useful to incorporate information about periodic volatility patterns and macroeconomic announcements in their calculations. Their article detailed these volatility and jump estimation methods. 
In this paper, we introduce some Markov chain Monte Carlo (MCMC) meth- ods developed in the statistical literature and apply these methods to develop an stochastic volatility SV model for USD/GHS exchange rate.

\section{Methodology}

\section{Stochastic Volatility (SV) Model}

A volatility model is defined in terms of its first and second moment also referred to as the mean and variance equation.

$$
\begin{aligned}
y_{t} & =\sigma_{t} \varepsilon_{t} \\
\sigma_{t}^{2} & =\mu+\alpha y_{t-1}^{2}+\beta \sigma_{t-1}^{2} \\
y_{t} & =100^{*} \ln \left(X_{t} / X_{t-1}\right)
\end{aligned}
$$

Where $X_{t}$ is the daily exchange prices, $y_{t}$ is the daily return with standard deviation $\sigma_{t}, \sigma_{t}^{2}$ is the volatility of the returns.

The model described above is a general autoregressive conditional heteroskedastic (GARCH) model subject to the parameter restrictions, $\mu>0, \alpha \geq 0, \beta \geq 0$, and $\alpha+\beta<1$.

A stochastic volatility model (SV) is an extension of the GARCH model, but instead of assuming a deterministic time-varying volatility evolution as in GARCH, the SV model assumes a stochastic timevarying volatility evolution. Thus the SV model is defined as:

$$
\begin{aligned}
y_{t} & =\sigma_{t} \varepsilon_{t} \\
\sigma_{t}^{2} & =\mu+\alpha y_{t-1}^{2}+(\beta-1) \sigma_{t-1}^{2}+\sigma_{t} \eta_{t}
\end{aligned}
$$

where $\varepsilon_{t} \sim$ iid $N(0,1)$ the $\eta_{t} \sim$ iid $N(0,1), \varepsilon_{t}$ and $\eta_{t}$ are independent. Taylor (1986), proposed the SV method to make volatility dependent on a latent, unobservable state variable $h_{t}$ defined by:

$$
\text { In } \sigma_{t}^{2}=h_{t}
$$

$h_{t}$ is referred to as $\log$ volatility of $y_{t}$. Replacing $\sigma_{t}$ by $\exp \left(h_{t} / 2\right)$, the SV model becomes a logvolatility model and is written in a state space form:

$$
\begin{aligned}
& y_{t}=\exp \left(h_{t} / 2\right) \varepsilon_{t}, \varepsilon_{t} \sim \operatorname{iid} N(0,1), \quad \mathrm{t}=1, \ldots, \mathrm{T} \\
& h_{t}=\mu+\phi\left(h_{t-1}-\mu\right)+\sigma_{t} \eta_{t} \sim \text { iid } N\left(0, \sigma_{\eta}^{2}\right), \quad \mathrm{t}=1, \ldots, \mathrm{T} \\
& h_{0} \sim N\left(\mu, \sigma_{\eta}^{2} /\left(1-\phi^{2}\right)\right)
\end{aligned}
$$

The parameter vector is $\theta=\left(\mu, \phi, \sigma_{\eta}\right)$; the level of $\log$-variance $\mu$, the persistence of $\log$-variance $\varphi$, and the volatility of log-variance $\sigma_{\eta}$. The initial condition for the state, $h_{0}$, are given by the unconditional mean and variance of $h_{t}$, i.e. $\mu$ and $\left.\sigma_{\eta}^{2} /\left(1-\phi^{2}\right)\right)$ respectively.

To complete the model setup, a prior distribution for the parameter vector $\theta$ needs to be specified. Following Kim et al. (1998), we choose independent components for each parameter, i.e. $p(\theta)=$ $p(\mu) p(\phi) p\left(\sigma_{\eta}\right)$.

A Markov Chain Monte Carlo algorithm is employed to estimate the parameters in a Bayesian context as well as the volatilities of the SV model due to the intractability of the likelihood function of the SV model. 


\section{Markov Chain Monte Carlo (MCMC)}

MCMC is a class of methods in which we can simulate draws that are slightly dependent and are approximately from a (posterior) distribution of the desired random variables; in our case with the latent $\log$-variances $\mathbf{h}$ and the parameter vector $\theta$. We then take those draws and calculate quantities of interest for the (posterior) distribution.

In Bayesian statistics, there are generally two MCMC algorithms that we use: the Gibbs Sampler and the Metropolis-Hastings algorithm.

\section{Gibbs Sampling}

Given $p(\theta)=p(\mu) p(\phi) p\left(\sigma_{\eta}\right)$, the Gibbs sampler is used to sample from the joint distribution if we know the full conditional distributions for each parameter. For each parameter, the full conditional distribution is the distribution of the parameter conditional on the known information and all the other parameters: $p\left(\theta_{j} \mid \theta_{-j}, \mathrm{y}\right)$ Suppose we have a joint density $f(x, y)$, the Hammersley-Clifford Theorem proves that the joint density can be written in terms of the conditional densities $f(x \mid y)$, and $f(y \mid x)$. The theorem shows that knowledge of the conditional densities allows us to get the joint density.

\section{Steps to Calculating Full Conditional Distributions}

Suppose we have a posterior $p(\theta \mid h)$. To calculate the full conditionals for each $\theta$, do the following:

1. Write out the full posterior ignoring constants of proportionality.

2. Pick a block of parameters (for example, $\mu$ ) and drop everything that doesn't depend on $\mu$.

3. Use your knowledge of distributions to figure out what the normalizing constant is (and thus what the full conditional distribution $p\left(\mu \mid \phi, \sigma_{\eta}, h\right)$.

4. Repeat steps 2 and 3 for all parameter blocks.

\section{Gibbs Sampler Steps}

Let's suppose that we are interested in sampling from the posterior $p(\theta \mid h)$, where $\theta$ is a vector of three parameters, $\mu, \phi, \sigma_{\eta}$

The steps to a Gibbs Sampler

1. Pick a vector of starting values $\theta^{(0)}$ (Defining a starting distribution $\Pi^{(0)}$ and drawing $\theta^{(0)}$ from it).

2. Start with any parameter (order does not matter, but start with $\mu$ for convenience). Draw a value $\mu^{1}$ from the full conditional $p\left(\mu \mid \phi^{(0)}, \sigma_{\eta}{ }^{(0)}, h\right)$.

3. Draw a value $\varphi^{(1)}$ (again order does not matter) from the full conditional $p\left(\phi \mid \mu^{(0)}, \sigma_{\eta}{ }^{(0)}, h\right)$. Note the use of the updated value of $\mu^{(1)}$.

4. Draw a value $\sigma_{\eta}{ }^{(1)}$ from the full conditional $p\left(\sigma_{\eta} \mid \mu^{(1)}, \phi^{(1)}, h\right)$ using both updated values. (Steps 2-4 are analogous to multiplying $\Pi^{(0)}$ and $\mathbf{P}$ to get $\Pi^{(1)}$ and then drawing $\theta^{(1)}$ from $\Pi^{(1)}$ )

5. Draw $\theta^{(2)}$ using $\theta^{(1)}$ and continually using the most updated values.

6. Repeat until we get $M$ draws, with each draw being a vector $\theta^{(t)}$.

Our result is a Markov chain with a bunch of draws of $\theta$ that are approximately from our posterior. We can do Monte Carlo Integration on those draws to get quantities of interest. 


\section{Empirical Results}

This section entails an analysis of the daily exchange rate performance of the Ghana Cedi to the American Dollar (\$), using data available from January, 2007 to April, 2016. The statistical computing tool employed for this work is R software.

\section{Statistics and Data Analysis}

First, we begin the analysis of the study by exploring various statistical properties of the data under consideration. Descriptive statistics displayed by Table 1 gives statistical summary of the daily log returns of Ghana Cedi (GHC) - US Dollar (USD) exchange rates collected between January 18, 2007 and April 18, 2016. The summary indicates an average of $\log$ returns to be 0.0000000 with standard deviation of 0.0090766. Also the distribution of the data has skewness of 0.46 which is approximately symmetric and pecked with positive kurtosis of 17.65 .

Table 1: log returns of GHC-USD exchange rates (January, 2007 April, 2016).

\begin{tabular}{|ll|}
\hline \multicolumn{2}{|l}{ SUMMARY } \\
MEASURES & GHC-USD EXCHANGE RATE \\
\hline MINIMUM & -0.0785100 \\
MAXIMUM & 0.1001000 \\
MEDIAN & -0.0005884 \\
MEAN & 0.0000000 \\
STANDARD DEVIATION & 0.0090766 \\
RANGE & 0.1786263 \\
SKEWNESS & 0.46 \\
KURTOSIS & 17.65 \\
\hline
\end{tabular}

Figure 1 gives a visual time series representation of the exchange rate dataset. Its subfigures show the price and $\log$ returns plots of the dataset respectively. The time series plot of the price has a diurnal smooth exponential behavior but exhibits fluctuations at latter years around 2014 to 2015. This somewhat unstable trend could be attributed to the economic instability experienced in Ghana during that period with other related factors. Now, we consider the results obtained by modelling the exchange rate.

\section{Stationarity Test for Exchange rate}

We consider the Level Stationarity by employing the KPSS Test with the hypotheses stated below:

$$
\begin{aligned}
& H_{o}: \text { Level Stationary } \\
& H_{a} \text { : Not Stationary }
\end{aligned}
$$

With a p-value of 0.01, the null hypothesis for the KPSS test was rejected. This implies that the series is not stationary. After differencing once, the series for Exchange rate became stationary.

\section{Modelling the Exchange rate Data}

In this section, we setup our stochastic volatility (SV) model for analysis in the exchange rate dataset considered for this study. The SV model that represents the volatility process (log-variance process) for a $\log$-returns $y_{t}$ is given by:

$$
\overline{\mathrm{y}}_{t}=e^{h_{t} / 2} \varepsilon_{t} \varepsilon \sim N(0,1)
$$




$$
h_{t}^{2}=\mu+\phi\left(h_{t-1}^{2}-\mu\right)+\sigma \eta_{t} \quad \eta \sim N(0,1)
$$
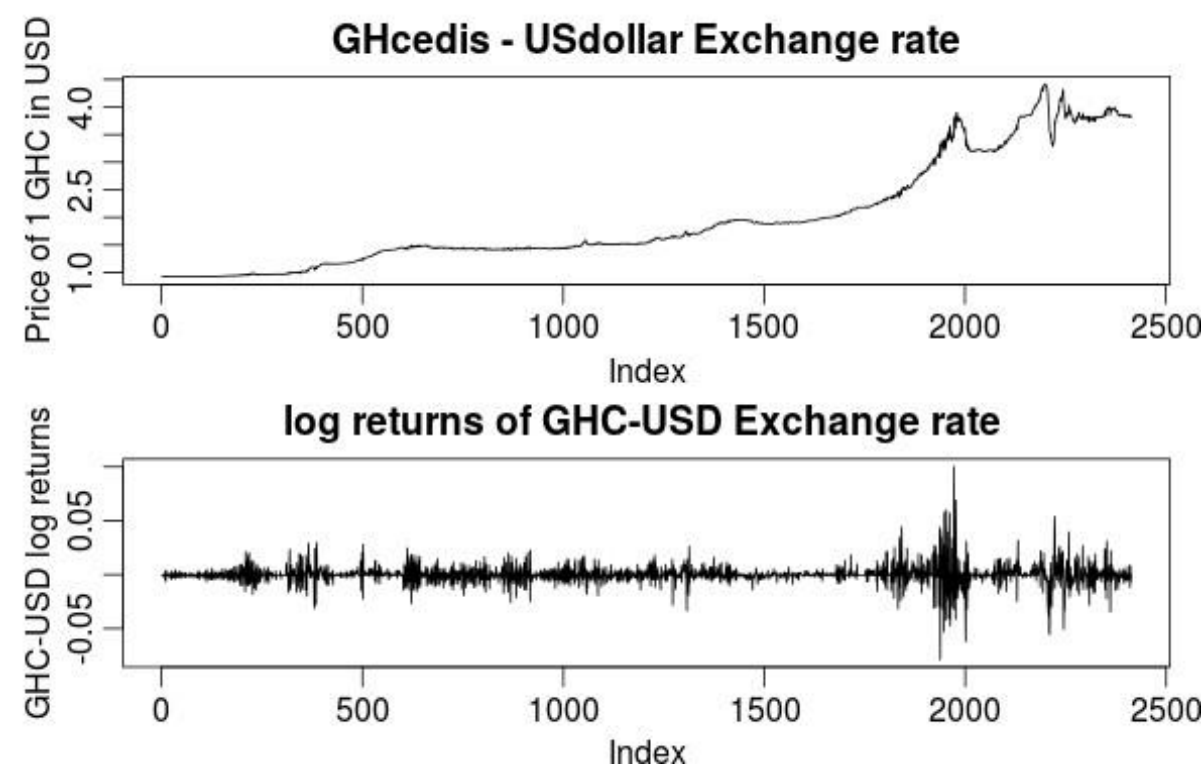

Figure 1: Visualization of time series plot of GHC -USD exchange rate and its log returns over the years between January, 2016 and April, 2016.

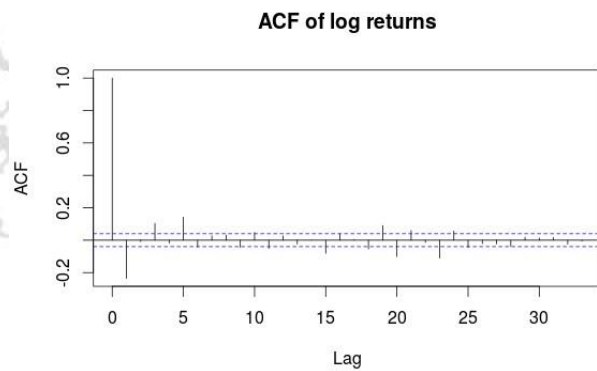

(a)

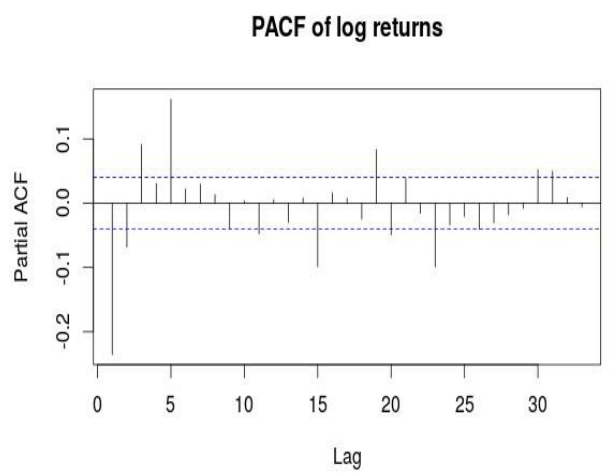

(c)

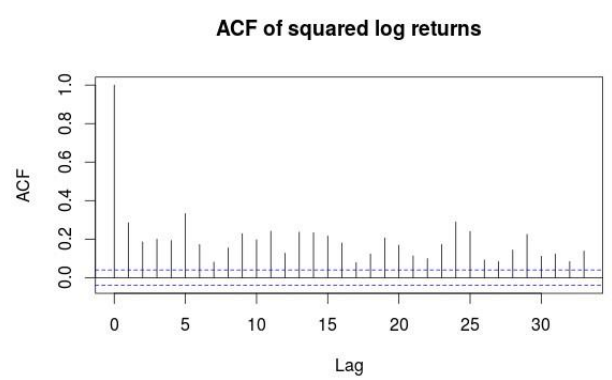

(b)

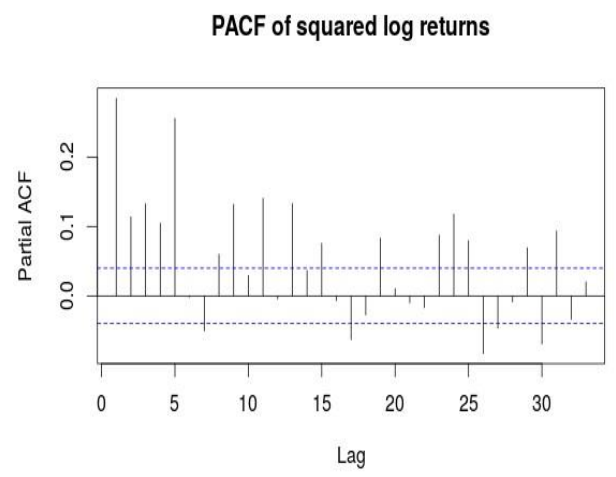

(d)

Figure 2: Autocorrelation (ACF) and Partial Autocorrelation (PACF) of log returns and squared log returns where $h=\left(h_{0, \ldots}, h_{t}\right)$ is the volatility process (log-variance process) and $\mu=-10.8320, \phi=0.9285$ and $\sigma=$ 0.6600 are considered to be level, persistence and the volatility of log-variance process respectively with 
their corresponding values can be observed from Table 2. The table shows the corresponding estimates of these parameter involved in the model. The estimates were obtained using stochval package in R. As described in our methodology, these estimates can be done using the Bayesian inference method mainly the Markov Chain Monte Carlo (MCMC) with a prior distribution of the stated parameters.

Table 2: parameter estimates of the SV model for the exchange rate dataset.

\begin{tabular}{|lcccccc|}
\multicolumn{7}{c|}{ POSTERIOR DRAWS OF PARAMETERS (thinning = 1) } \\
$\mu$ & Mean & std. dev & $5 \%$ & $50 \%$ & $95 \%$ & ESS \\
$\Phi$ & -10.8320 & 0.18973 & -11.1403 & -10.8323 & -10.5162 & 6357 \\
$\Sigma$ & 0.9285 & 0.01170 & 0.9086 & 0.9290 & 0.9465 & 401 \\
$e^{\mu / 2}$ & 0.6600 & 0.04542 & 0.5865 & 0.6588 & 0.7387 & 214 \\
$\sigma^{2}$ & 0.0045 & 0.00043 & 0.0038 & 0.0044 & 0.0052 & 6357 \\
\hline
\end{tabular}

Figure 3 show gives the posterior quantiles plot of the volatilities in percent which is the empirical quantiles of the posterior distribution of the log returns over time. It observed that despite these range of quantile percentage, the estimated volatilities pictorially looks closer to each other. We then make forecast of 100 step-ahead volatility prediction and that is made evidence with the dotted lines in figure 3 . Figure 4 displays a combined plots of other quantiles and the trace of the three parameters considered in the formulation of the stochastic volatility model with their symmetric density distributions.

From figure 5, it is observed that the Q-Q plot for the mean standardized residuals has most of its residuals falling on the line or not deviating from the line of best fit and its distribution looks approximately normal. And this provides the assessment.

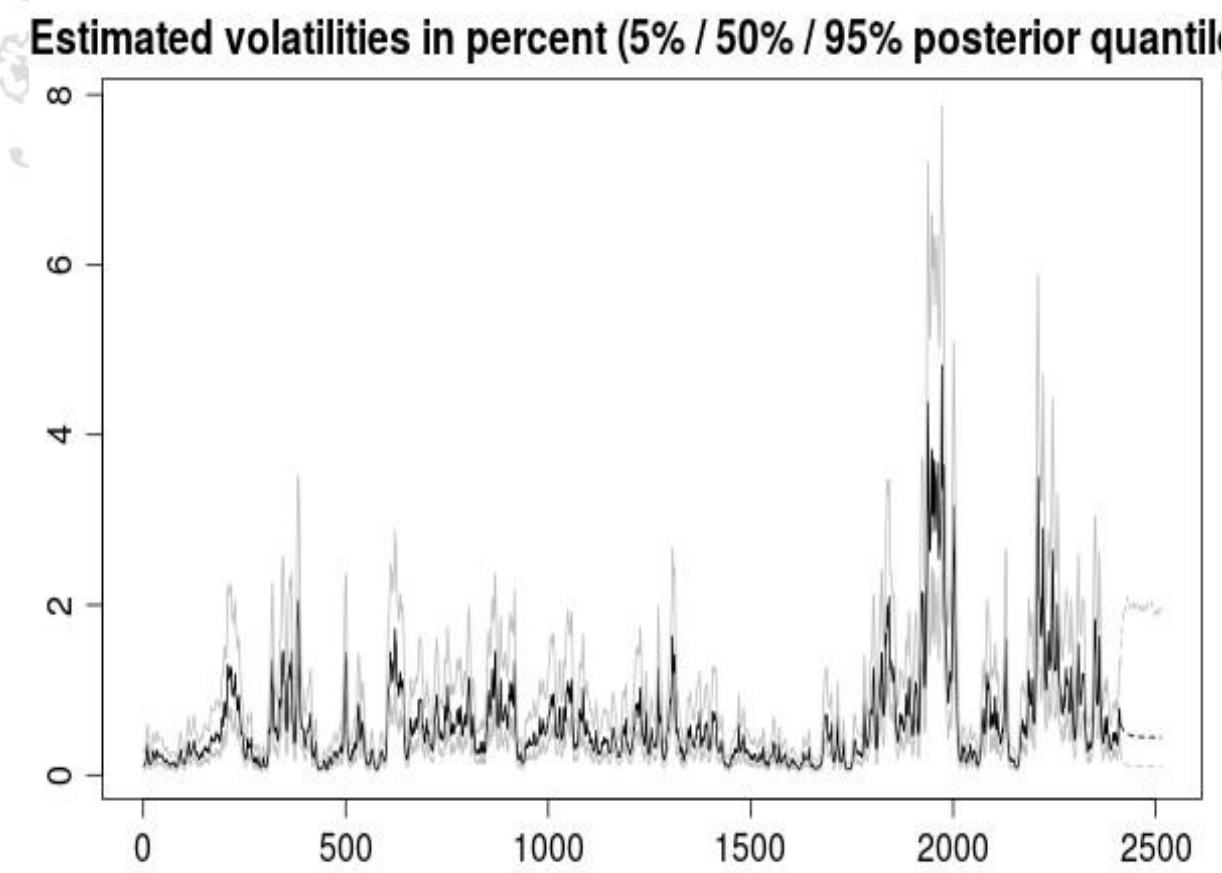

Figure 3: estimated volatilities of GHC-USD exchange rate with predicted future volatilities show as the dotted lines. 

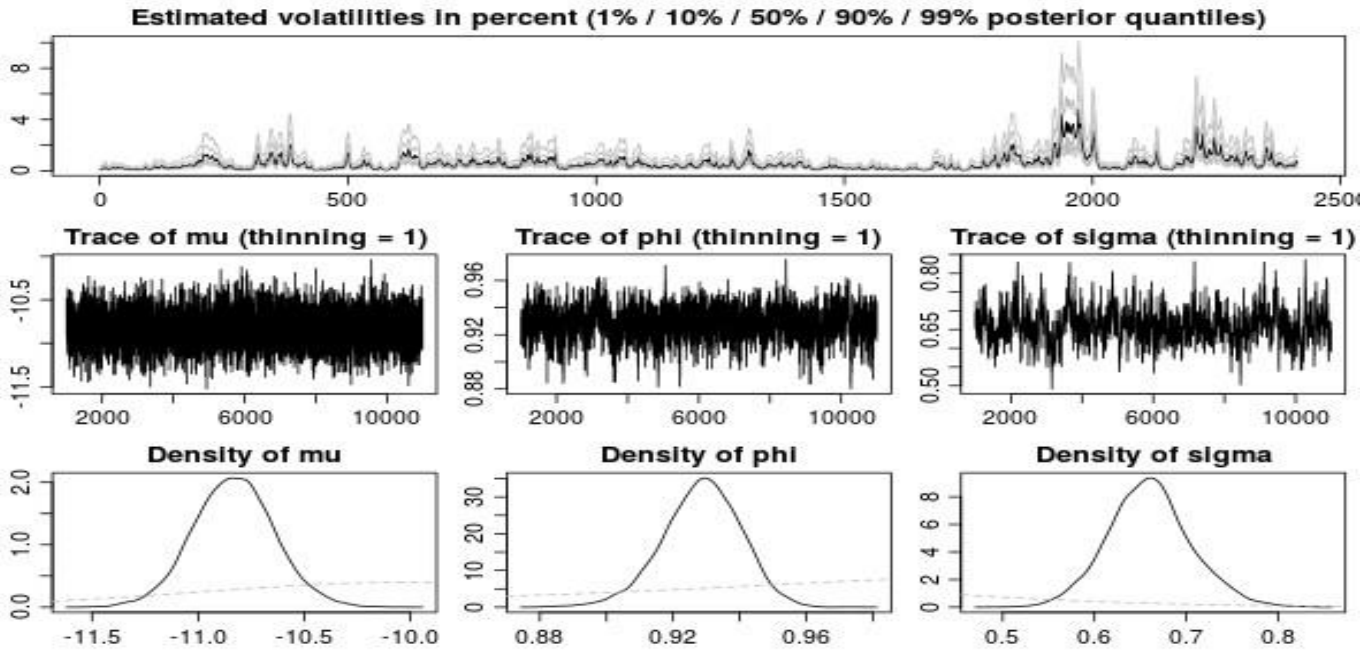

Figure 4: This plot shows: (1) the other percentage quantiles with median shown as the black line (2) trace plots for the parameters in the model and (3) the posterior density estimates (black solid lines) along with prior densities (dashed gray lines).
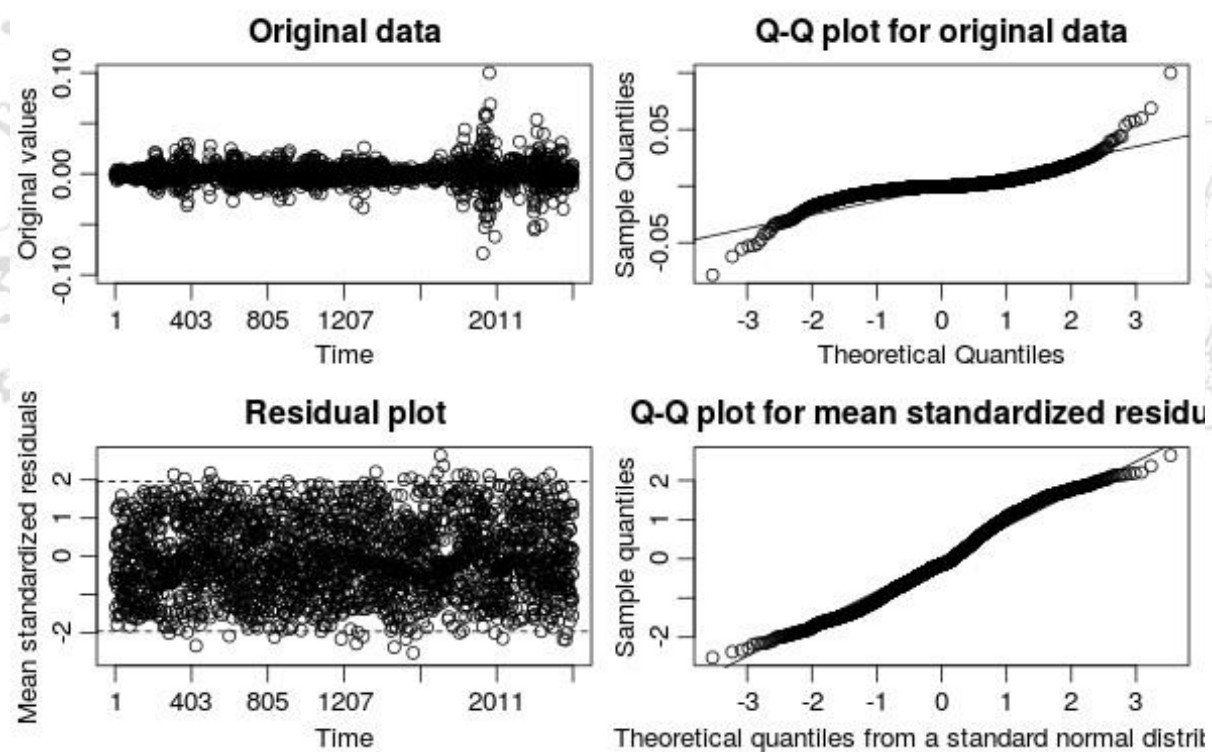

Figure 5: Visualization of the Q-Q plot of the original data and for the mean standardized residuals

\section{Conclusion}

This paper examined the application of stochastic volatility of returns on the Ghana Cedi and US dollar (\$) exchange rate. The preliminary analysis of data used reveals the Stationarity after one time differencing of dataset. To ascertain the volatility, we proposed the use of stochastic volatility model as an application in estimating and forecasting the volatilities of the log-returns of GHC-USD exchange rate dataset collected between the years of (January, 2007 to April, 2016). The Bayesian inference method mainly, the Markov Chain Monte Carlo (MCMC) was used in estimating the parameters of the model. It was observed from our figures that period of high volatilities were mostly in the latter part of the returns series. This could be attributed to slowdown of growth since during the years of 2013-14, GDP growth fell to a low of 3.5\%. 
And also, due to speculative trading in the currency markets. It is also observed that the estimated volatility plots given by the posterior quantiles are quite close together before the predicted future values which is due to the smooth exponential behavior of prices of the GHC - USD exchange rate. Finally, the study recommends the use and comparison of other variant models such as GARCH model in estimation of volatility.

\section{References}

Byun, S. K., Rhee, D. W. and Kim, S. (2011) "Intraday volatility forecasting from implied volatility", International Journal of Managerial Finance, Vol. 7 Iss: 1, pp.83 - 100

Cayen, J-P., Coletti, D., Lalonde, R., and Maier, P. (2009), "What Drives Exchange Rates? New Evidence from a Panel of U.S. Dollar Bilateral Exchange Rates”, www.ecb.europa.eu

Erdemlioglu, D., Laurent, S. and Neely, C.J. (2013) "Econometric Modeling of Exchange Rate Volatility and Jumps", Federal Reserve Bank of St. Louis Working Paper 2013-024.

Frimpong, J.M. and Oteng-Abayie, E.F. (2006). "Modelling and Forecasting Volatility of Returns on the Ghana Stock Exchange using GARCH Models", Munich personal RePEc Archive, 593, 1-21.

Hamao, Y., Ronald W.M., Victor N. (1990), "Correlations in Price Changes and Volatility across International Stock Markets”, The Review of Financial Studies 1990 Volume 3, number 2. pp. 281-30

Harvey, S. K., Adjasi, C. and Agyapong, D. (2008), "Effect of exchange rate volatility on Ghana stock exchange", African Journal of Accounting, Economics, Finance and Banking Research. Vol.3. No.3.

Ishida, I., McAleer, M. and Oya, K., (2011). "Estimating the Leverage Parameter of Continuous -time Stochastic Volatility Models Using High Frequency SnP 500 and VIX", http://eprints.ucm.es

Kim, S., Shephard, N., and Chib, S. (1998), "Stochastic Volatility: Likelihood Inference and Comparison with ARCH Models," The Review of Economics Studies, 65, 361-393.

Melino, A., and Turnbull, S. (1990), "P ricing Foreign Currency Options With Stochastic Volatility,". Journal of Econometrics, 45, 7-39.

Taylor, S.J. (1986), “Modelling Financial Time Series”. Chichester, UK. 